\title{
Accidents vasculaires des patients âgés : ne baissez pas les bras !
}

\author{
Marc Verny \\ C Springer-Verlag France 2010
}

Pourquoi avoir choisi de consacrer le dossier thématique de ce nouveau numéro des Cahiers de l'année gérontologique aux accidents vasculaires cérébraux (AVC) des sujets âgés ? On pourrait répondre : parce qu'il y a urgence, et ce, sans mauvais jeu de mots autour de la prise en charge de l'AVC à sa phase aiguë !

En effet, comme le montre très bien l'article de Yannick Béjot, les chiffres parlent d'eux-mêmes et caractérisent ce que l'on a l'habitude de qualifier de « vrai problème de santé publique »... Et il s'agit bien d'une problématique gériatrique, puisque plus de la moitié des AVC surviennent chez des plus de 70 ans. L'augmentation de cette tranche de la population est rapide et on risque donc d'être confronté à une augmentation en valeur absolue de cette pathologie dans cette tranche d'âge. Pourtant, l'analyse des données épidémiologiques présentées donne quelques raisons d'espérer : dans le grand âge, ce sont surtout les lacunes dont l'incidence progresse et donc un risque moindre de séquelle majeure, et l'âge de survenue du premier AVC a reculé de cinq ans. On vit donc plus longtemps sans faire d'AVC.

Le grand paradoxe est que si l'AVC des patients âgés est fréquent et grave (première cause de handicap et $25 \%$ de mortalité à un mois chez les 80 ans et plus), on en parle finalement assez peu, et la capacité pour la personne âgée ou son entourage d'identifier des symptômes ou des signes en rapport avec un AVC est très mauvaise. Pourtant, le temps est un facteur déterminant dans la qualité de la prise en charge. Des efforts sont clairement à poursuivre dans ce domaine avec des campagnes d'éducation qui devraient voir le jour prochainement, incitant à appeler le « 15 » lors d'une symptomatologie évocatrice. Il ne faudra pas oublier de sensibiliser la population âgée de manière spécifique d'autant que les patients âgés sont souvent réticents à venir à l'hôpital. Le problème est d'autant plus difficile que, comme nous le rappelle Sandrine Greffard, la sémiologie

\footnotetext{
Marc Verny $(\square)$

Centre de gériatrie, pavillon M.-Bottard, Hôpital de la Salpêtrière, AP-HP et UPMC, 47-83, boulevard de l'Hôpital, F-75651 Paris cedex 13, France e-mail : marc.verny@psl.aphp.fr
}

révélatrice peut être extrêmement atypique dans cette tranche d'âge, et parfois seul le clinicien expérimenté saura repérer la suspicion d'AVC « au milieu du reste ». C'est en tout cas dans ces situations que l'IRM cérébrale se révèle performante. Elle le sera même en cas de négativité permettant de réorienter la démarche diagnostique et donc la prise en charge thérapeutique. Par ailleurs, l'IRM permet de préciser l'état des artères intracérébrales, l'existence de lésions hémorragiques et apporte donc des informations majeures pour la prise en charge des patients comme nous le rappelle Emmanuel Ellie. Le bilan permettra aussi de mettre en évidence, le plus souvent, le mécanisme et la cause de l'AVC. Dans $25 \%$ des cas, il mettra même en évidence des causes potentielles multiples, et il faudra choisir la bonne thérapeutique avec comme objectif principal : éviter la récidive!

Mais enfin me direz-vous, tout cela a-t-il un sens et ne faut-il pas les laisser tranquilles, car on sait bien que l'on ne pourra pas faire grand-chose et dans le fond, s'ils ne veulent pas venir à l'hôpital ils n'ont peut-être pas tort!

Voilà encore des a priori qui n'ont pas de raison d'être, comme nous le montrent très bien les articles d'Yves Guilhermet, d'Hélène Mahagne, d'Yves Samson et de Mathieu Züber. Tout tourne autour d'une prise en charge précoce et spécialisée qui permet non seulement de réduire la mortalité, mais aussi (et surtout à cet âge ?) de réduire les séquelles fonctionnelles. Le travail sur le type de structure, l'organisation des soins et la fluidité des filières sont aussi déterminants pour tenter d'offrir à tous une prise en charge optimale. Cela est d'autant plus vrai que l'accès aux UNV des patients âgés à très âgés reste encore difficile et pourtant rien ne justifie cela, puisque cette population bénéficie tout à fait de cette prise en charge, à condition d'être autonome au moment de l'AVC. Les données montrent aussi que la population âgée bénéficie tout à fait d'une endartériectomie carotidienne pour sténose symptomatique, mais à condition d'une intervention rapide après la survenue de l'infarctus et de confier le patient à une équipe entraînée à faible taux de complications chirurgicales. Le bénéfice serait même plus important chez les plus de 75 ans : que demande le gériatre ? Que 1'on puisse thrombolyser ses patients ? Pourquoi pas ? 
Là encore, rien ne semble authentiquement s'y opposer, mais il faut encore pouvoir affiner les critères pour définir la population qui bénéficiera réellement de cette technique. La démarche réadaptative permet quant à elle de réduire le retentissement fonctionnel des déficits, et c'est bien la raison d'être d'une équipe pluridisciplinaire autour de ces patients (kinésithérapeutes, ergothérapeutes, orthophonistes, assistant social...).

Et le fonctionnement cognitif ? C'est certainement, comme nous le rappelle Olivier Godefroy, le point noir de l'évolution à distance d'un $\mathrm{AVC}$ avec un risque important de survenue de démence, qu'il s'agisse d'une authentique démence vasculaire ou d'un trouble mixte, d'un dysfonctionnement cognitif de novo ou de l'aggravation de troubles préexistants. Il faut, en tous cas, être très attentif à cela et effectuer une évaluation cognitive dans les trois à six mois qui suivent l'AVC.

Ainsi, comme tous ces articles nous le montrent, il n'y aucune raison ni justification à une attitude défaitiste chez les patients âgés qui présentent un AVC. L'âge ne saurait être une limite en soi ! Tout doit être fait pour offrir aux patients âgés une prise en charge optimale avec un accès aux explorations et aux thérapeutiques les plus performantes et les plus modernes. À ces heures de grande tentation de restriction des soins, il faut avoir conscience qu'en l'occurrence, le coût d'un patient âgé avec un lourd handicap est bien plus élevé que celui d'un bilan et d'une prise en charge thérapeutique adaptée et efficiente. Cela est plus coûteux sur le plan médicoéconomique et bien plus encore sur le plan humain ! À l'inverse, il faut savoir rapidement identifier les patients pour lesquels un processus de dépendance était déjà engagé avant l'AVC, car dans ces cas, il faudra savoir adapter les bilans et les traitements. Ces décisions difficiles doivent pouvoir être prises en concertation et nous ne pouvons que plaider pour une large coopération entre neurovasculaires et gériatres pour le plus grand bénéfice de nos patients.

Bonne lecture ! 